3. J. L. Ericksen and R. A. Toupin, Implications of Hadamard's condition for elastic stability with respect to uniqueness theorems, Canadian Journal of Mathematics vol. 8 (1956) pp. 432-436.

4. A. V. Biçadze, On the uniqueness of solutions of the Dirichlet problem for elliptic partial differential equations, Uspekhi Matematičeskih Nauk (N.S.) vol. 3 (1948) pp. 211-212.

Naval Research Laboratory, Washington, D. C.

\title{
ON THE IDENTITIES OF CERTAIN ALGEBRAS
}

\section{ADIL YAQUB}

Introduction. One basic problem in the study of finite algebras is concerned with the existence of a finite basis for the identities of the algebra, i.e., a finite set of identities from which all the identities of the algebra are logical consequences. That the identities of a finite algebra need not have a finite basis (in the above sense) has already been observed by Lyndon [3]. This leads naturally to the following question: are there certain classes of finite algebras the identities of which possess a finite basis? We shall answer this question in the affirmative. Indeed, the main result of this paper is the following

TheOREM. A functionally strictly complete algebra which contains more than one element has a finite basis for its identities.

1. Preliminary concepts. In this section, we shall review some basic concepts and definitions, all of which are to be found in Foster $[1 ; 2]$.

Let $\mathfrak{A}=(A, \rho, \cdots)$ be a universal algebra with primitive operations $\rho, \cdots$. Let $A=\{\cdots, x, \cdots\}$.

A (k-ary) 2-function $f\left(x_{1}, \cdots, x_{k}\right)$ is a composition, via the primitive operations, of indeterminate symbols $x_{1}, \cdots, x_{k}$ over the set $A$ together with a (possibly empty) set of $\operatorname{constants}^{1}$ ( $=$ fixed $\in A$ ).

An $\mathfrak{A}$-function is called strict if it involves no constants.

In an obvious way each $\mathfrak{A}$-function $f\left(x_{1}, \cdots, x_{k}\right)$ represents (or has associated with it) a mapping of the set $A^{k}$ into $A$, where of course different $\mathfrak{A}$-functions need not represent different such mappings. If $\mathfrak{A}$-functions $f(x, \cdots)$ and $g(x, \cdots)$ represent the same mapping we

Presented to the Society, April 14, 1956; received by the editors February 10, 1956 and, in revised form, August 25, 1956.

1 In more uniform terminology a constant may also be defined as a " 0 -ary" function. 
speak of an $\mathfrak{A}$-identity, $f(x, \cdots)=g(x, \cdots)$. If both $f$ and $g$ are strict $\mathfrak{A}$-functions we speak of a strict $\mathfrak{A}$-identity.

$\mathfrak{A}$ is finite, of order $n$, if $A$ is a class of $n$ elements.

$\mathfrak{A}$ is said to be (functionally) complete-respectively (functionally) strictly complete-if $A$ is finite and if each mapping of the set $A \times \cdots \times A$ into $A$ may be expressed as some $\mathfrak{A}-$ function-respectively as some strict $\mathfrak{A}$-function. For examples and criteria of functional completeness see $[1 ; 2]$.

2. The finite basis theorem. In preparation for this theorem, we recall the following result which was proved in [2] and which we shall state as a lemma.

Lemma 1. Let $\mathfrak{U}, \overline{\mathfrak{U}}$ be any universal algebras of the same species and each containing at least two elements, and where

(a) $\mathfrak{U}$ is finite and functionally strictly complete.

(b) Each strict identity of $\mathfrak{U}$ is also an identity of $\overline{\mathfrak{u}}$. Then

(i) $\overline{\mathfrak{U}}$ is isomorphic with a subdirect sum of $\mathfrak{u}$.

(ii) $\mathfrak{U}$ and $\overline{\mathfrak{U}}$ satisfy precisely the same strict identities.

This is an immediate combination of Theorem 9.1, Theorem 9.2 and Theorem 6.2 of [2].

Although in the above lemma we assumed that each strict identity of $\mathfrak{u}$ is also an identity of $\overline{\mathfrak{u}}$, in the proof of this lemma only a finite number of strict $\mathfrak{U}$-identities were assumed to be $\overline{\mathfrak{U}}$-identities (see $[1 ; 2]$ ). Let this finite set of strict $\mathfrak{U}$-identities be denoted by $I$. It is now fairly evident that the above lemma can be strengthened as follows:

Lemma 1'. Same as Lemma 1 except that hypothesis (b) is now replaced by the weaker hypothesis

$\left(b^{\prime}\right)$ Each strict identity of $\mathfrak{U}$ in the set $I$ is also an identity of $\overline{\mathfrak{u}}$.

We are now in a position to prove the following important

THEOREM 1. Let $\mathfrak{U}$ be a functionally strictly complete algebra of order $n \geqq 2$. Then the strict identities of $\mathfrak{U}$ have the above finite set $I$ of strict $\mathfrak{U}$-identities as a finite basis.

Proof. Let $\mathfrak{B}$ be any algebra which satisfies set $I$ and let $L$ be any strict identity of $\mathfrak{U}$. Theorem 1 will be proved by showing that $L$ is also a strict identity of $\mathfrak{B}$. But this follows, since if $\mathfrak{B}$ is a one-element algebra, $\mathfrak{B}$ obviously satisfies $L$, while if $\mathfrak{B}$ has order greater than 1 , $\mathfrak{B}$ again satisfies $L$ by Lemma $1^{\prime}$ (conclusion (ii)). This proves the theorem. 
We shall conclude the paper by referring to Lyndon's example [3]. It is of course to be noted that his algebra is not strictly functionally complete. This is easily seen from Theorem 1 above, and is indeed clear by inspection.

In conclusion, I wish to express my gratitude and indebtedness to Professor A. L. Foster for his generous counsel.

\section{REFERENCES}

1. A. L. Foster, Generalized "Boolean" theory of universal algebras, Part I, Math. Zeit. vol. 58 (1953) pp. 306-336.

2. - Generalized "Boolean" theory of universal algebras, Part II, Math. Zeit. vol. 59 (1953) pp. 191-199.

3. R. C. Lyndon, Identities in finite algebras, Proc. Amer. Math. Soc. vol. 25 (1954) pp. 8-9.

\section{Purdue University}

